\title{
VIEWPOINT
}

\section{Physician administered sedation for DC cardioversion}

\section{S J Harrison, J Mayet}

Providing anaesthetic cover for DC cardioversion can sometimes prove a challenge for the cardiologist, with potentially disastrous consequences for the patient

$\mathrm{E}$ xternal DC (direct current) cardioversion for atrial tachyarrhythmias is a common cardiological procedure that has traditionally been performed with the patient under general anaesthesia. However organising anaesthetic cover for a cardioversion can sometimes be a challenging experience for the cardiologist. He/she often has to coordinate their own availability with: a starved patient, a coronary care nurse, an anaesthetist, and a trained anaesthetic assistant. This process is time consuming and may be frustrating for staff and patients when delays are encountered, and can lead to longer hospital stays with cost implications. Physicians in a variety of specialities have experience in providing safe sedation for unpleasant procedures in accordance with the many guidelines now available. ${ }^{1}$ In cardiology the widespread use of transoesophageal echocardiography has led to familiarity in the administration of sedative drugs. ${ }^{2}{ }^{3}$

In response to the difficulties in arranging anaesthetic cover there has been a resurgence of interest in physician administered sedation for external DC cardioversion. Two groups have recently described their experiences in this journal using benzodiazepines with opiate supplementation as required, and have concluded that this was a safe and effective method of performing cardioversions. ${ }^{45}$ These studies were performed with appropriate monitoring and resuscitation equipment for sedation and report no serious adverse events. However, the studies were underpowered to provide reassurance for this technique (149 and 141 patients enrolled), and raise a number of concerns.

\section{LEVEL OF SEDATION}

The first issue relates to the level of sedation required for this procedure. External DC cardioversion may be a very brief procedure, but is profoundly stimulating and needs anaesthesia and not merely sedation to obtund stress responses effectively and prevent recall. The pain level for external cardioversion can be equated to that of electroconvulsive therapy-which is of a similar brief duration and for which a short general anaesthetic is also given. Electrophysiology procedures and transoesophageal echocardiography are lengthier, and sedation is to reduce discomfort and allay anxiety rather than to produce anaesthesia. In the two recent studies published in this journal an unusually deep level of sedation was induced to prevent recall, as indicated by loss of eyelash reflex ${ }^{4}$ or a Ramsay sedation score of $5^{5}$ (described as a sluggish response to light glabellar tap or loud auditory stimulus). ${ }^{6}$ In fact, most of the patients in these studies could be described as being in the light planes of anaesthesia. This distinction has also been highlighted in the Practice guidelines for sedation and analgesia by non-anaesthesiologists. ${ }^{7}$ They define sedation and analgesia as a state that allows patients to tolerate unpleasant procedures while maintaining adequate cardiorespiratory function and the ability to respond purposefully to verbal command and/or tactile stimulation. They suggest that patients whose only response is reflex withdrawal from a painful stimulus are sedated to a greater degree than encompassed by sedation/analgesia-in other words, they are anaesthetised.

This leads on to the second concern, which is one of airway safety. It is in the light planes of anaesthesia that airway difficulties are most commonly encountered such as laryngospasm, coughing, bronchospasm, and subsequent hypoxia. Laryngospasm results from a predominance of adductor muscle tone of the vocal cords. If severe-with complete apposition of the vocal cords-there will be complete airway obstruction. This can usually be overcome by skilled airway management, and with either deepening or lightening the level of anaesthesia, but on occasion specific anaesthetic drugs such as suxamethonium (a fast acting muscle relaxant which breaks the spasm) are required. It is the unpredictability of heavy sedation/light anaesthesia that makes anaesthetists reluctant to offer sedation without a trained anaesthetic assistant and full anaesthetic equipment at hand. In fact it is often safer to opt for anaesthesia from the start and guarantee better airway control, especially in children, smokers, the obese, and patients with obstructive sleep apnoea.

\section{HYPERCAPNIA}

A third concern is that of hypoventilation and carbon dioxide retention. Intravenous anaesthetic induction agents, benzodiazepines, and opioids all cause dose related respiratory depression and eventually apnoea. Pulse oximetry measuring oxygen saturation is a monitor of hypoxia, but provides no information as to the level of arterial carbon dioxide. It is easy for inadequate ventilation (and therefore a climbing $\mathrm{PaCO}_{2}$ ) to be masked if the patient has supplementary oxygen. It is not suggested that supplementary oxygen should not be used, as avoiding hypoxia is vital. However, it should be recognised that when a longer duration and deeper level of sedation are used patients are likely to become significantly hypercapnoiec, which may in itself precipitate 
arrhythmias and deepening coma. In anaesthetic practice, spontaneously breathing anaesthetised patients routinely have their end tidal carbon dioxide levels monitored, and management instigated to prevent dangerous levels of hypercapnia developing.

The routine use of flumazenil to reverse benzodiazepine is worthy of comment. Patients run the risk of becoming resedated when the half life of the benzodiazepines given outlasts that of flumazenil. ${ }^{89}$

Although superficially attractive, benzodiazepines with or without opiates are not the ideal drugs for use in external DC cardioversion. An intravenous induction agent such as propofol is a much better choice because it has rapid onset, obtunds laryngeal reflexes well (lowering the incidence of laryngospasm), but redistributes quickly and so has a rapid offset obviating the need for reversal. ${ }^{10}{ }^{11}$ Common to most anaesthetic/sedative agents, propofol does produce dose dependent hypotension more notably in elderly and dehydrated patients, but this can be limited by slower administration and careful titration in more susceptible patients.

In conclusion, the elective cardiovascularly stable patient for external DC cardioversion needs to be deeply sedated or anaesthetised. Conscious sedation is only appropriate for other less painful procedures. The new report on Implementing and ensuring safe sedation practice ${ }^{12}$ is very clear in its recommendations on conscious sedation. It states that if verbal responsiveness is lost the patient requires a level of care identical to that needed for general anaesthesia.

The problem is that anaesthetic/sedative emergencies are relatively rare and it is easy to be lulled into a false sense of security. Emergencies, when they do occur, can lead to serious consequences if not dealt with using appropriate speed by personnel with appropriate airway skills. Cardiologists should work with their anaesthetic colleagues to get more readily available anaesthetic cover for these procedures rather than offering a suboptimal service that may lead to the occasional disaster. Regular, formal day case lists with appropriate anaes--thetic and recovery staff is the ideal solution, with flexibility from all concerned to accommodate urgent cases.

\section{Authors' affiliations}

S J Harrison, Department of Anaesthetics, Lister Hospital, Stevenage, Herffordshire, UK

J Mayet, Peart-Rose-Waller Centre for Circulatory Health, St Mary's Hospital, Imperial College, London, UK

\section{REFERENCES}

1 Working Party Report. Guidelines for sedation by non-anaesthetists. London: Royal College of Surgeons of England, 1993.

2 Saltissi M, de Belder MA, Nihoyannopoulos P. Setting up a transoesophageal echocardiography service. Br Heart J 1994;71/suppl 4): 15-19.

3 Sutaria N, Northridge D, Denvir M. A survey of sedation and monitoring practices during transoesophageal echocardiography in the UK: are recommended guidelines being followed? Heart 2000;84(suppl II):ii 19.

4 Pugh PJ, Spurrell P, Kamalvand K, et al. Sedation by physician with diazepam for DC cardioversion of atrial arrhythmias. Heart 2001;86:572-3

5 Raipancholia R, Sentinella L, Lynch M. Role of conscious sedation for external cardioversion. Heart 2001;86:571-2.

6 Ramsay MAE, Savage TM, Simpson BR, et al. Controlled sedation with alphaxalone-alphadalone. BM 1974;2:656-9

7 American Society of Anesthesiologists Task Force on Sedation and Analgesia by Non-Anesthesiologists. Practice guidelines for sedation and analgesia by non-anesthesiologists. Anesthesiology 1996:84:459-71.

8 Daniel PD, Robert SH, Thomas HW. Reversal of midazolam-induced laryngospasm with flumazenil. Ann Emerg Med 1998;32:263-5.

9 Jones RD, Chan K, Roulson CJ, et al. Pharmacokinetics of flumazenil and midazolam. Br J Anaesth 1993;70:891-9.

10 Stoneham MD. Anaesthesia for cardioversion. Anaesthesia 1996:51:565-70.

11 Bryson HM, Fulton BR, Faulds D. Propofol. An update of its use in anaesthesia and conscious sedation. Drugs 1995;50:513-59.

12 UK Academy of Medical Royal Colleges and their Faculties. Implementing and ensuring safe sedation practice for healthcare procedures in adults. Nov 2001. Report of an Intercollegiate Working Party chaired by the Royal College of Anaesthetists. www.aomrc.org.uk/ publications.htm.

\section{STAMPS IN CARDIOLOGY}

\section{Quinine}

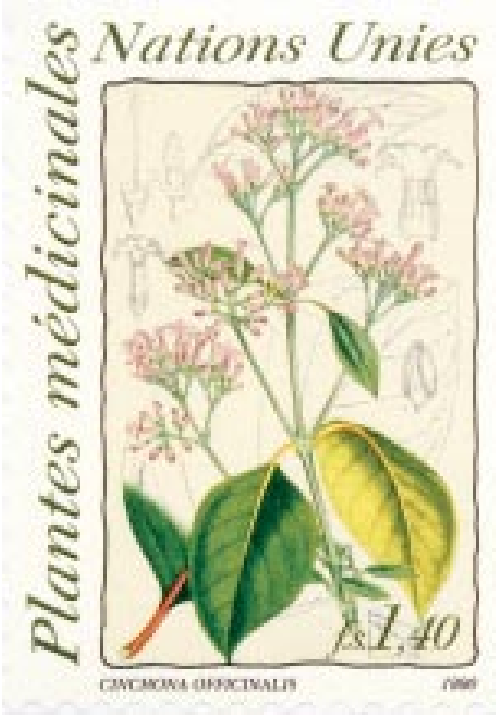

Cinchona officinalis (family Rubiaceae) is a tree from the Andes whose bark contains the alkaloids quinine and quinidine. "Jesuit's bark", as it was called, was discovered in Europe after 1630 to be valuable in treating malaria. It also became widely used for fevers in general, and in 1749 de Senac found by chance that "Long and rebellious palpitations have ceded to this febrifuge". The alkaloids had been isolated by 1860 but their proven use in arrhythmias came about only because of the astute observation in 1912 of a Dutchman with attacks of palpitation who had found for himself that he could halt an attack when he took $1 \mathrm{~g}$ of quinine. Probably he took quinine for malaria. He was a patient of Professor Karel Wenckebach who confirmed paroxysmal atrial fibrillation with an ECG and related the episode later (JAMA 1922;81:472-4). Wenckebach often tried quinine but succeeded in only one other patient. Then W von Frey in Berlin studied all four cinchona alkaloids and found that quinidine was the most effective. In 1920 Thomas Lewis pioneered the use of chest leads to record precisely the atrial rate in atrial fibrillation and showed that it was slowed by quinidine. Referring to his hypothesis of circus movement Lewis proposed that normal rhythm was restored because quinidine closed the gap between the crest and the wake of the circus wave.

Cinchona belongs to the huge family Rubiaceae that has over 10000 species worldwide, but emetine from ipecacuanha and caffeine from coffee are the only other drugs within it. It contains the beautiful shrub Gardenia named by Linnaeus to honour the Scotsman Dr Alexander Garden who was a physician and botanist in Charleston, South Carolina around 1780.

The stamp showing Cinchona officinalis came from the United Nations (Geneva Headquarters) in 1990 as part of the set depicting medicinal plants. 